\title{
Natural Orifice Specimen Extraction (NOSE) with Single- Stapling Anastomosis for Left Colon Cancer
}

\author{
Ming-Yin Shen, M.D., Ph.D., William Tzu-Liang Chen, M.D. \\ Department of Colorectal Surgery, China Medical University Hsinchu Hospital, Hsinchu, Taiwan
}

\begin{abstract}
One of the major criticisms of laparoscopic colectomy is the requirement of an additional mini laparotomy to remove the resected specimen. This may be associated with postoperative pain and wound complications. Therefore, the use of a natural orifice as a delivery route for the specimen extraction without a laparotomy incision can subsequently reduce the risk of wound complications. In this video, we demonstrate the natural orifice specimen extraction procedure with a side-to-end single-stapling colorectal anastomosis.
\end{abstract}

Keywords: Laparoscopy, Colorectal neoplasm, Natural orifice specimen extraction, Surgical anastomosis

Supplementary video file: This article contains supplementary material (https://doi.org/10.7602/ jmis.2020.23.4.201).

This is an Open Access article distributed under the terms of the Creative Commons Attribution Non-Commercial License (http:/ creativecommons.org/licenses/by-nc/4.0/) which permits unrestricted non-commercial use, distribution, and reproduction in any medium, provided the original work is properly cited.

\author{
Received February 3, 2020 \\ Revised 1st April 17, 2020 \\ 2nd July 26,2020 \\ Accepted October 16, 2020 \\ Corresponding author \\ Ming-Yin Shen \\ Department of Colorectal Surgery, \\ China Medical University Hsinchu \\ Hospital, No. 199, Sec. 1, Xinglong \\ Rd., Zhubei City, Hsinchu County \\ 302, Hsinchu 30272, Taiwan \\ Tel: +886-3-5580-558 \\ Fax: +886-3-5580-018 \\ E-mail: mingyin.shen@gmail.com \\ ORCID: \\ https://orcid.org/0000-0003-0978-908X
}

Copyright $\odot 2020$ The Journal of Minimally Invasive Surgery. All rights reserved.

\section{INTRODUCTION}

Laparoscopic colorectal surgery is currently considered as a golden standard for surgical treatment of colorectal cancer. ${ }^{1}$ However, one of the major problems is the additional abdominal incision required for specimen retrieval. The additional wound may result in postoperative pain, surgical site infection, or incisional hernia. Therefore, natural orifice specimen extraction (NOSE) via the anus or vagina was used to mitigate these unfavorable outcomes. The purpose of this video is to show the feasibility of the NOSE operation by providing a step-by-step demonstration of the procedure. This procedure of NOSE was approved by the Ethics Advisory Committee of China Medical University Hospital before initiation and was registered in the
CMUH Clinical Trials Registry as CMUH102-REC2-133.

\section{PROCEDURE}

The same as the conventional laparoscopic colectomy, the patients lay supine in the reverse Trendelenburg and right down tilting position. After completion of the inferior mesenteric artery and inferior mesenteric vein ligation, medial to lateral mesocolic dissection with or without splenic flexure mobilization, and pelvic dissection, which were the same as those in the conventional procedure, we performed NOSE with a completely intracorporeal colonic resection, followed by transanal specimen extraction and side-to-end single-stapling colorectal anastomosis (see details in the video and below). 
After division of the mesorectum at the distal resection margin, the lumen of the rectum was occluded by a free tie, and then the rectal stump was irrigated. The rectum and proximal colon were divided using an energy device.

To minimize the risk of tumor implantation during the NOSE procedure, a) the extraction site was dilated with a largediameter rectal dilator, b) the extraction site was lubricated with jelly, and c) the transrectal route was protected by an extra-small Alexis wound protector (Applied Medical, CA, USA) inserted transanally and positioned in the rectum.

By the introduction of a Babcock clamp, the specimen was retrieved from the anus. Forceful attempts at extraction may result in specimen perforation or stump trauma and should be avoided. If the delivery of the specimen is difficult, the NOSE procedure should be aborted and converted to conventional modes of specimen extraction. Anvil head was introduced through the anus and was fixed to the side wall of colon by purse-string suture. Then, the colonic stump was closed with an endoscopic linear cutter stapler, and the wound protector was removed. After an intracorporeal purse-string suture was made around the rectal stump, side-to-end single-stapling colorectal anastomosis was performed using an intraluminal circular stapler.

\section{RESULTS}

From December 2018 to December 2019, sixteen patients underwent laparoscopic NOSE with single-stapling side to end colorectal anastomosis for left colon cancer in the Department of Colorectal Surgery of China Medical University Hsinchu Hospital, Taiwan. Median operating time was 222 (191 250) min. No conversion was required. The average of estimated blood loss (EBL) was 14 milliliters. The median hospital stay was 5 (range 4 9) days. None of these patients has postoperative complication.

\section{DISCUSSION}

One randomized trial reported better short-term surgical outcomes, including reduced pain and lower analgesia requirements, for laparoscopic NOSE colectomy than for the conventional laparoscopic colectomy. ${ }^{2}$ A recently published study from our institution demonstrated the safety and feasibility of NOSE-AR (Anterior Resection) by comparing 82 patients who underwent NOSE AR for sigmoid or upper rectal cancers with those who underwent conventional AR. ${ }^{3}$ The patients who underwent NOSE-AR had significantly lower rates of postoperative opioid use, lesser hospital stay duration, and faster return of bowel movement. Other variables such as $\mathrm{R} 0$ resection rate, operative duration, and blood loss were equivalent.

In our NOSE laparoscopic surgery, we intended to obtain better surgical outcomes by using a side-to-end single-stapling colorectal anastomosis. Compared with the double-stapling anastomosis technique, the purse-string suture closure of the rectal stump may prevent further distal rectal stump dissection. The single-stapling technique also prevents the formation of "dog ears," thus possibly eliminating the risk of anastomosis leakage. In addition, side-to-end colorectal anastomosis may have better blood perfusion and functional outcome than straight anastomosis.

Based on our previous experience, the keys to the success of NOSE-AR with a side-to-end single-stapling colorectal anastomosis include proper selection of the patients, good bowel preparation, tension free anastomosis, adequate blood perfusion, and reinforcement of anastomosis as well. For the majority of the patients, complete splenic flexure mobilization is required to obtain an adequate bowel length for a tension free anastomosis. Executing a complete purse-string suture over the distal rectum is the other important technical tip, so that the stapler can catch the complete circumference of the rectum. We also assess blood perfusion by intraoperative fluorescence angiography with Indocyanine green (ICG). In addition, the anastomosis is reinforced by sutures, and is checked by intraoperative colonoscopy.

No gold standard surgical approach has been established for laparoscopic NOSE surgery, but in this study, we demonstrated a feasible and safe NOSE procedure with a side-to-end singlestapling colorectal anastomosis for left colon cancer.

\section{ORCID}

Ming-Yin Shen, https://orcid.org/0000-0003-0978-908X

William Tzu-Liang Chen,

https://orcid.org/0000-0002-6585-2437

\section{AUTHORS' CONTRIBUTIONS}

Conceptualization: William Tzu-Liang Chen. Formal analysis: Ming-Yin Shen. Methodology: Ming-Yin Shen, William TzuLiang Chen. Writing-original draft: Ming-Yin Shen. Writingreview and editing: William Tzu-Liang Chen.

\section{CONFLICT OF INTEREST}

None.

\section{FUNDING}

None.

\section{ACKNOWLEDGMENTS}

None. 


\section{REFERENCES}

1) Jayne DG, Guillou PJ, Thorpe H, et al. Randomized trial of laparoscopic-assisted resection of colorectal carcinoma: 3-year results of the UK MRC CLASICC Trial Group. J Clin Oncol 2007;25:3061-3068.

2) Wolthuis AM, Fieuws S, Van Den Bosch A, de Buck van Overstraeten A, D'Hoore A. Randomized clinical trial of laparoscopic col- ectomy with or without natural-orifice specimen extraction. Br J Surg 2015;102:630-637.

3) Saurabh B, Chang SC, Ke TW, et al. Natural Orifice Specimen Extraction With Single Stapling Colorectal Anastomosis for Laparoscopic Anterior Resection: Feasibility, Outcomes, and Technical Considerations. Dis Colon Rectum 2017;60:43-50. 